\title{
Divergence of Function and Regulation of Class B Floral Organ Identity Genes
}

\author{
Alon Samach, ${ }^{a}$ Susanne E. Kohalmi, ${ }^{b, 1}$ Patrick Motte, ${ }^{c}$ Raju Datla, ${ }^{d}$ and George W. Haughn a,2 \\ a Botany Department, University of British Columbia, 3529-6270 University Boulevard, Vancouver, British Columbia, \\ V6T 1Z4, Canada \\ ${ }^{b}$ Biology Department, University of Saskatchewan, Saskatoon, Saskatchewan, S7N 0W9, Canada \\ c Max-Planck-Institut für Züchtungsforschung, Carl-von-Linne Weg 10, D-50829 Cologne, Germany \\ d Plant Biotechnology Institute, National Research Council of Canada, 110 Gymnasium Road, Saskatoon, \\ Saskatchewan, S7N OW9, Canada
}

Regulatory mechanisms controlling basic aspects of floral morphogenesis seem to be highly conserved among plant species. The class B organ identity genes, which are required to establish the identity of organs in the second (petals) and third (stamens) floral whorls, are a good example of such conservation. This work compares the function of two similar class B genes in the same genetic background. The DEFICIENS (DEF) gene from Antirrhinum, including its promoter, was transformed into Arabidopsis and compared in function and expression with the Arabidopsis class $B$ genes APETALA3 (AP3) and PISTILLATA (PI). The DEF gene was expressed in the second, third, and fourth whorls, as was PI. Functionally, $D E F$ could replace $A P 3$ in making petals and stamens. The DEF gene's $A P 3$-like function and $P I$-like expression caused transformation of fourth-whorl carpels to stamens. Like $A P 3$, all aspects of $D E F$ function in Arabidopsis required a functional PI protein. Surprisingly, DEF could not replace the AP3 protein in properly maintaining AP3 transcripts (autoregulation). Our data allow us to revise the current model for class $B$ autoregulation and propose a hypothesis for the evolution of class B gene expression in dicotyledonous plants.

\section{INTRODUCTION}

Antirrhinum and Arabidopsis are distantly related dicotyledonous species (occupying different subclasses of Dicotyledoneae) with similar but distinct floral morphologies. The analysis of floral homeotic mutants in both species has permitted the identification and characterization of many regulatory genes that direct floral morphogenesis. Surprisingly, on the basis of recessive mutant phenotypes, amino acid sequence identity, and patterns of transcript distribution, the set of floral regulatory genes identified in Arabidopsis is almost identical to the set identified in Antirrhinum. Thus, the regulatory mechanisms controlling basic aspects of floral morphogenesis seem to be highly conserved (reviewed in Davies and Schwarz-Sommer, 1994; Ma, 1994; Weigel and Meyerowitz, 1994; Haughn et al., 1995).

Among the best-studied floral regulatory genes are the class $B$ organ identity genes, which are required in part to establish the identity of organs in the second (petal) and third (stamen) floral whorls. Two class B genes are known for both Antirrhinum (DEFICIENS [DEF] and GLOBOSA [GLO]) and Arabidopsis (APETALA3 [AP3] and PISTILLATA [PI]). LosS-of-

'Current address: Plant Sciences Department, Biological-Geological Sciences Building, University of Western Ontario, 1151 Richmond Street North, London, Ontario, N6A 5B7, Canada.

${ }^{2}$ To whom correspondence should be addressed. E-mail haughn@ unixg.ubc.ca; fax 604-822-6089. function mutations in any one of the four genes result in similar homeotic transformations: petals to sepals and stamens to carpels (Bowman et al., 1989, 1991; Hill and Lord, 1989; Schwarz-Sommer et al., 1990, 1992; Sommer et al., 1990; Tröbner et al., 1992). All four genes have been cloned, and their nucleotide sequences were determined (Sommer et al., 1990; Jack et al., 1992; Tröbner et al., 1992; Goto and Meyerowitz, 1994; Okamoto et al., 1994; Irish and Yamamoto, 1995). Each of the genes contains a MADS box DNA binding domain (Schwarz-Sommer et al., 1990) and a K-box (potential dimerization domain; Ma et al., 1991). On the basis of the deduced amino acid sequence, AP3 is closely related to DEF (61.2\% sequence identity) and PI is related to GLO $(58.4 \%$ sequence identity). In contrast, the promoters of the $A P 3$ and $D E F$ genes are significantly divergent.

The expression of the Arabidopsis and Antirrhinum class $B$ transcripts and protein products has been studied extensively (Schwarz-Sommer et al., 1990, 1992; Jack et al., 1992, 1994; Tröbner et al., 1992; Goto and Meyerowitz, 1994; Zachgo et al., 1995; Krizek and Meyerowitz, 1996a). For all four class $B$ genes, transcripts are initially detected in young flower primordia at the time of sepal initiation (stage 3; stages as defined by Smyth et al., 1990; Zachgo et al., 1995), but there are differences in their spatial distribution. One of the two class $B$ genes from each organism is transcribed in the 
second-, third-, and fourth-whorl primordial cells (DEF and $P I$ ), whereas transcripts of the second class $B$ gene are limited mainly to the second- and third-whorl primordial cells (GLO and AP3). The Arabidopsis and Antirrhinum class B genes expressed in fourth-whorl primordial cells (DEF and $P I)$ are not considered to be orthologous genes on the basis of amino acid sequence similarity. At later stages, class $B$ gene transcripts are maintained at a high level only in the second- and third-whorl primordial cells of developing floral shoots, with transcripts persisting in developing petals and stamens until the completion of flower development. However, low levels of transcript of some of the class $B$ genes have been detected in first-whorl (DEF and $A P 3$ ) and fourthwhorl (DEF) organs of flowers in later stages of development. Class $B$ proteins become detectable in the second- and third-whorl primordial cells at stage 4, when sepal primordia have become separated from the floral apex and start to overgrow it (Jack et al., 1994; Zachgo et al., 1995). Class B proteins have not been found in the first- or fourth-whorl organ primordia.

The complexity of the expression pattern of class B genes suggests that they are highly regulated. Indeed, several genes are known to be required for this process (Schultz et al., 1991; Bowman et al., 1992; Weigel and Meyerowitz, 1993; Simon et al., 1994; Ingram et al., 1995; Levin and Meyerowitz, 1995; Sakai et al., 1995; Wilkinson and Haughn, 1995). Functional class $B$ proteins are themselves required to maintain class $B$ gene expression in developing second- and third-whorl organs (Schwarz-Sommer et al., 1992; Tröbner et al., 1992; Goto and Meyerowitz, 1994; Jack et al., 1994).

Genetic, molecular, and biochemical studies with class B genes from both species have helped us to understand some aspects of how the class B organ identity genes function. Early in floral development (at the time of sepal initiation), $D E F$ and GLO genes in Antirrhinum and $A P 3$ and $P /$ genes in Arabidopsis are transcribed in overlapping domains (Sommer et al., 1990; Jack et al., 1992; Schwarz-Sommer et al., 1992; Tröbner et al., 1992; Goto and Meyerowitz, 1994; Krizek and Meyerowitz, 1996a). In the region of overlap (second- and third-whorl primordial cells), the two different class $B$ polypeptides specifically interact to form a heterodimeric protein complex (Schwarz-Sommer et al., 1992; Tröbner et al., 1992; Goto and Meyerowitz, 1994; Davies et al., 1996a; Riechmann et al., 1996; S.E. Kohalmi and W.L. Crosby, unpublished results). Formation of the heterodimer protein stabilizes the class B polypeptides (Jack et al., 1994; Zachgo et al., 1995; Krizek and Meyerowitz, 1996a), allows entry into the nucleus (in Arabidopsis; McGonigle et al., 1996), and permits binding to MADS domain target sequences (CarG motifs; Schwarz-Sommer et al., 1992; Tröbner et al., 1992; Riechmann et al., 1996). Targets of the heterodimeric class $B$ protein might include the promoters of the class $B$ genes themselves (autoregulation) because maintenance of class $B$ transcripts depends on the availability of a functional class B protein (Schwarz-Sommer et al., 1992; Tröbner et al., 1992; Goto and Meyerowitz, 1994; Jack et al., 1994; Zachgo et al.,
1995; Krizek and Meyerowitz, 1996a). The requirement of both proteins in a cell for both stability and autoregulation could explain why expression in the first or fourth whorls of just one of the pair is transient.

Despite the similarities between Arabidopsis and Antirrhinum class $B$ floral regulatory genes, a closer look at the putative class $B$ orthologous genes $A P 3$ and DEF shows that they differ in many ways. First, mutations in $D E F$ eliminate fourth-whorl organs; yet, in Arabidopsis class B mutants, fourth-whorl organs still develop (Jack et al., 1992; SchwarzSommer et al., 1992). Second, temperature shift assays with temperature-sensitive mutants show a difference in the time they are required for normal stamen development (Bowman et al., 1989; Zachgo et al., 1995). Third, initial transcript expression is different: $A P 3$ is expressed in the base of the sepals, whereas $D E F$ is initially expressed in the fourth whorl and is slightly expressed in sepals later in development (Jack et al., 1992, 1994; Schwarz-Sommer et al., 1992). Finally, although $D E F$ expression depends on a functional GLO protein, transcriptional regulation of $A P 3$ in second-whorl organs seems to be independent of $P I$ function (Schwarz-Sommer et al., 1992; Goto and Meyerowitz, 1994).

There is still little direct evidence concerning the functional equivalence of putative orthologous floral regulatory genes in general and for $D E F$ and $A P 3$ in particular. We have introduced the $D E F$ gene into Arabidopsis and compared its function with that of $A P 3$. Our data show that $D E F$ can complement the strong ap3-3 mutation. Because complementation depends on the appropriate spatial and temporal expression of the DEF gene, the interaction of the DEF protein with $\mathrm{PI}$, and the activation of the appropriate target genes, our data strongly support the hypothesis that $D E F$ is orthologous to $A P 3$. This work also allows us to identify clear differences between the two genes, providing new insights on class B function, maintenance, and evolution.

\section{RESULTS}

\section{Transgenic Arabidopsis Plants Carrying the DEF Gene Show a Homeotic Floral Phenotype}

Our primary objective was to examine the effect of introducing the genomic Antirrhinum DEF gene (including its own promoter) into Arabidopsis. For this purpose, we cloned a 7-kb EcoRl genomic fragment from Antirrhinum containing the DEF gene (Schwarz-Sommer et al., 1992) into a binary transformation vector (see Methods) with selective markers for both kanamycin resistance and glucuronidase (GUS) activity. This construct was transformed into wild-type Arabidopsis $\left(T_{1}\right)$ by using Agrobacterium-mediated transformation. Three independent kanamycin-resistant $T_{2}$ transformants were examined. Kanamycin resistance and GUS activity segregated among the $T_{3}$ progeny at a frequency suggesting that each line carried three (TDF1) or one (TDF2 and TDF3) loci 
with a T-DNA. DNA gel blot analyses indicated that the transformants contained an intact DEF gene (data not shown) with a copy number of one (TDF3), two (TDF2), or approximately $\operatorname{six}(T D F 1)$.

Two of the three lines containing DEF, TDF1 and TDF2, had a floral phenotype distinct from the wild type that segregated with the kanamycin resistance marker. The phenotype, designated Tdef, was a homeotic transformation of the fourth-whorl carpels into stamens. Whereas the first emerging flowers were phenotypically similar to wild-type flowers, each successive flower displayed a stronger homeotic transformation than did the previous one. Figure 1 shows wild-type flowers and flowers with a typical Tdef phenotype from plant line TDF1. The earlier flowers were either indistinguishable from the wild type or had distinctly curved pistils (Figure 1B). Later flowers had gynoecia consisting of both stamen and carpel tissue fused together that resulted in split and distorted gynoecia (stamen-carpel organs; Figures $1 \mathrm{C}, 2 \mathrm{~J}$, and $2 \mathrm{~K}$ ). There was considerable variation in the ratio of stamen to carpel tissue in such flowers. Finally, for some of the latest emerging flowers in the inflorescence, the gynoecium was replaced by a variable number of stamens and stamen-carpel organs (Figure 1D), with the maximum number of extra stamens being four. Although all three floral phenotypes were seen in TDF1 and TDF2 plants, both the number of mutant flowers and the degree to which each flower was affected (expressivity) was higher in TDF1 than in TDF2.

To determine whether the severity of the phenotype was due to loci number, the TDF1 plant was outcrossed to the wild type to obtain lines segregating for only one locus. All single-loci lines showed a Tdef phenotype, proving that one locus of the DEF gene is enough to cause fourth-whorl transformations. One of these TDF1 derivative lines (TDF1-1), carrying two loci, was used in subsequent crosses. Significantly, the Tdef floral phenotype is similar to that of Arabidopsis floral morphogenesis mutant 10 (Flo10, also known as Superman) mutants or transgenic plants in which the AP3 gene is under the control of the cauliflower mosaic virus $35 \mathrm{~S}$ promoter (Schultz et al., 1991; Bowman et al., 1992; Jack et al., 1994). In both of these latter mutants, the stamen tissue in the fourth whorl has been correlated with and attributed to expression of $A P 3$ and $P I$ in cells of the fourth whorl. One major difference between the Tdef and Flo10 phenotypes is that as one moves along the inflorescence in an acropetal direction, the Tdef floral phenotype becomes more severe whereas the Flo10 phenotype becomes less severe (Bowman et al., 1992). Unlike Flo10 mutants (Gaiser et al., 1995), the Tdef floral phenotype did not include any changes in ovule development or structure.

\section{Expression of Class B Organ Identity Genes in Transgenic Arabidopsis Tdef Plants}

Transcripts of the class B organ identity genes of both Arabidopsis and Antirrhinum are present at high levels in cells
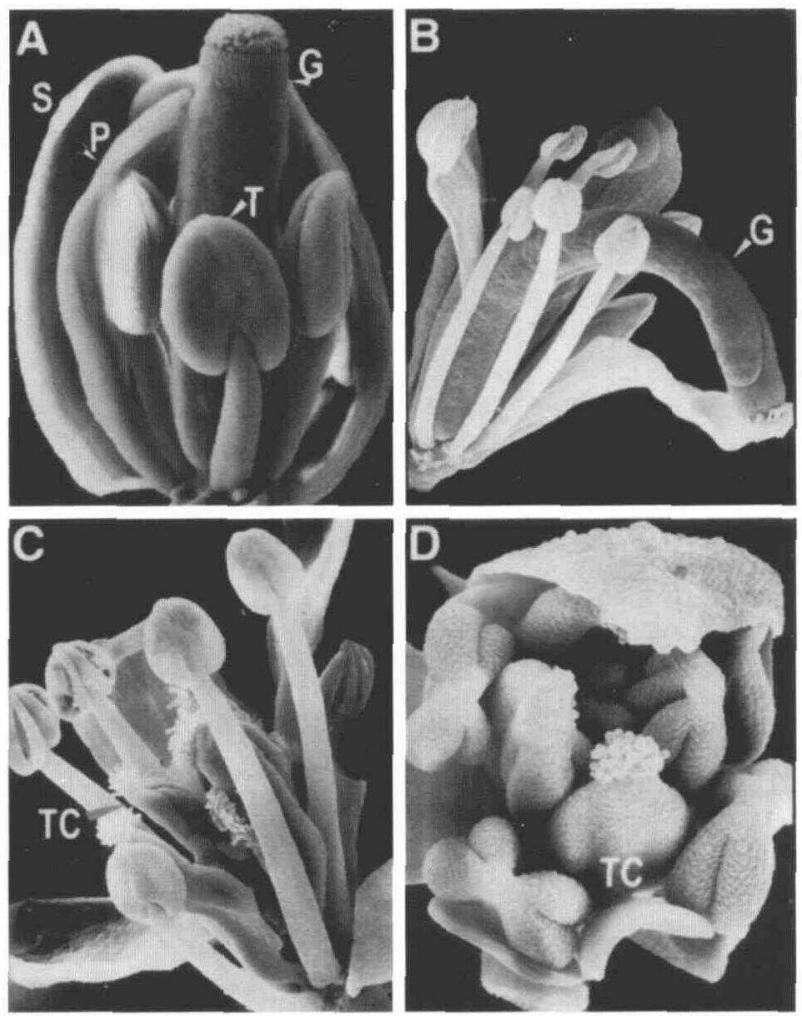

Figure 1. Scanning Electron Microscopy of Flowers from Transgenic Arabidopsis Plants Expressing the DEF Gene.

Some perianth organs were removed to show more clearly the reproductive organs.

(A) Wild-type flower with first-whorl sepals (S), second-whorl petals $(P)$, third-whorl stamens (T), and a fourth-whorl gynoecium (G). Magnification is $\times 34$.

(B) A flower from the TDF1 line showing a weak Tdef phenotype. Notice the distinctly curved gynoecium (G). Magnification is $\times 18$.

(C) A flower from the TDF1 line with fourth-whorl stamen-carpel organs (TC). Notice the stigmatic papillae on the fourth-whorl stamenlike organs. Magnification is $\times 26$.

(D) A flower from the TDF1 line in which the gynoecium is replaced by stamens and stamen-carpel organs (TC). Magnification is $\times 62$.

of the second- and third-whorl primordia and developing petals and stamens of wild-type plants (Jack et al., 1992, 1994; Schwarz-Sommer et al., 1992; Tröbner et al., 1992; Goto and Meyerowitz, 1994). In addition, DEF and PI transcripts are present in fourth-whorl primordial cells in young floral primordia until just before the emergence of petal and stamen primordia. In Flo10 mutants or transgenic Arabidopsis plants in which the AP3 gene is under the control of the cauliflower mosaic virus $35 \mathrm{~S}$ promoter, the class $B$ genes are also expressed in cells of the developing fourth whorl (Bowman et al., 1992; Goto and Meyerowitz, 1994; Jack et al., 1994). 

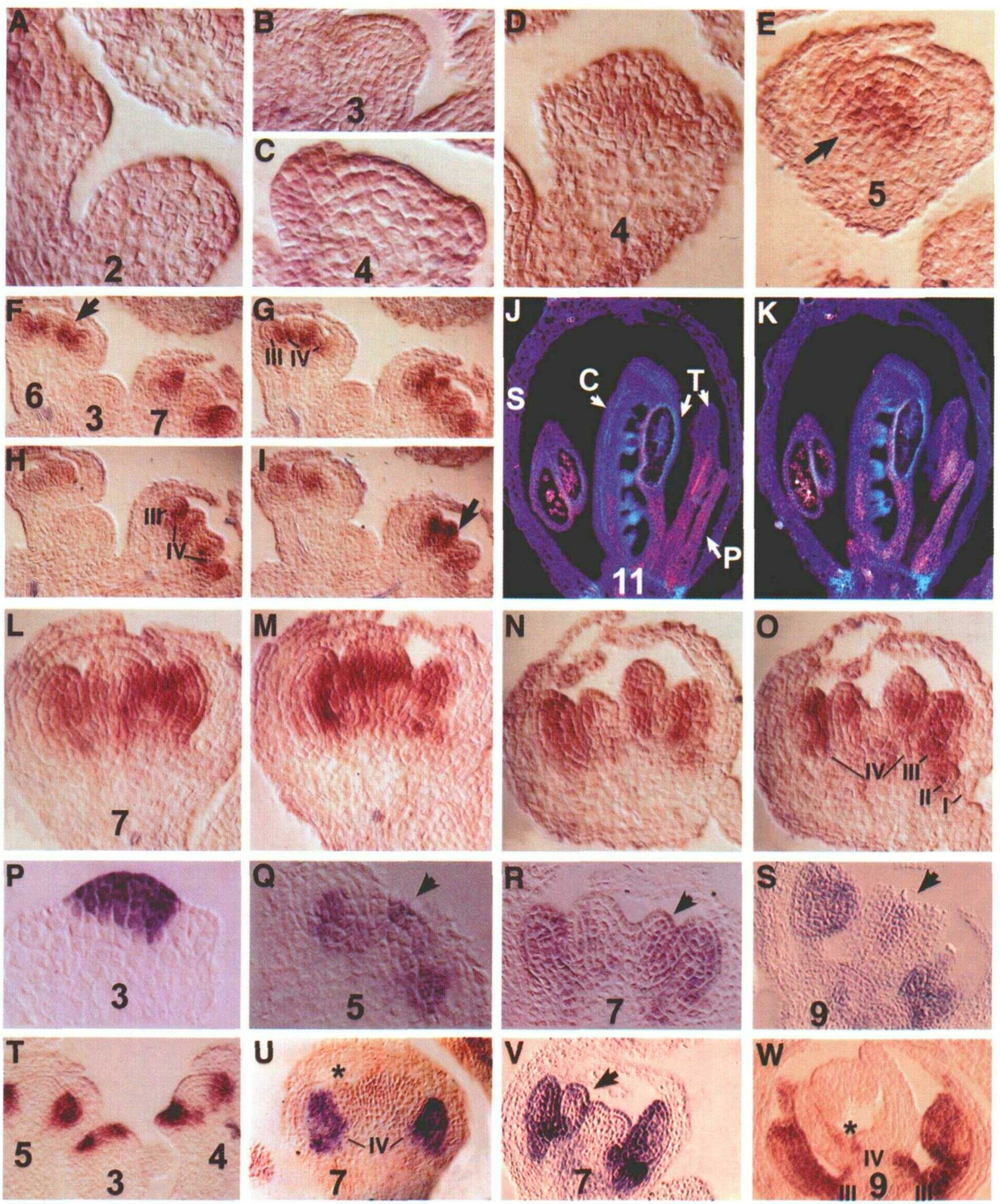

Figure 2. Distribution of Class B mRNAs in Inflorescences and Flowers of Transgenic TDF Plants.

Sections in $(\mathbf{J})$ and $(\mathbf{K})$ were hybridized with ${ }^{35}$ S-labeled antisense DEF RNA. The dark-field exposure, used to detect the silver grains, is superimposed on a UV fluorescence image to visualize the underlying tissue stained with calcofluor white. All other sections were hybridized with digoxigenin-labeled antisense RNA probes and photographed under differential interference contrast optics, with the transcript signal visible as dark brown or blue. All sections, except for the one shown in (V) (TDF2), are from TDF1 plants. All sections are longitudinal. 
In situ hybridization was used to determine the pattern of $D E F, A P 3$, and $P I$ transcripts in TDF1 and TDF2 plants (Figure 2). The DEF transcript was not detected in wild-type Arabidopsis flowers (control), indicating that under the hybridization conditions used, the $D E F$ probe was specific for the $D E F$ transcript (data not shown). In early stages of development of Tdef flowers (stages 2 to 4 ), no DEF transcript was detected above background levels (Figures $2 \mathrm{~A}$ to $2 \mathrm{D}$ ). The $D E F$ transcript was first clearly detected in early stage 5 flowers in the center of flower primordia (Figure 2E). Later in flower development, after the initiation of petal and stamen primordia, the $D E F$ probe detected transcripts in the developing second and third whorls of Tdef flowers of all stages (Figures $2 \mathrm{~F}$ to 2O). The signal within these primordia seemed less uniform than that normally found for $A P 3$ and $P I$ in wild-type flowers (Figures $2 \mathrm{~L}$ to $2 \mathrm{O}$ ). In addition, and consistent with the abnormal fourth-whorl phenotype of Tdef flowers, DEF was expressed in sectors of variable size in fourth-whorl primordia (Figures 2F to 2O). In mature flowers, such fourth-whorl expression was observed only in flowers with curved (small sectors) or staminoid (large sectors) fourth-whorl organs. In the latter type, the larger sectors containing DEF transcript always encompassed those parts of the gynoecium that were obviously staminoid (Figures $2 \mathrm{~J}$ and $2 \mathrm{~K}$ ).

The PI (Figures 2P to 2S) pattern of expression in secondand third-whorl organs of Tdef flowers was similar to that of the wild type. In wild-type flowers, expression in fourthwhorl primordial cells is transient, whereas in Tdef plants, $P$ I transcript was maintained in sectors of the developing fourth-whorl organs of most Tdef flowers (Figures $2 \mathrm{Q}$ to $2 \mathrm{~S}$ ). The AP3 (Figures 2T to $2 \mathrm{~W}$ ) pattern of expression in secondand third-whorl organs of Tdef flowers was also similar to that of wild-type plants. Unlike $P I$ and $D E F$, in most Tdef flowers, $A P 3$ transcripts were not detected in any fourthwhorl organs, even when they were clearly abnormal (Figure $2 U)$. In a few Tdef flowers ( $\sim 10 \%$ of flowers with abnormal fourth-whorl organs), AP3 transcripts were detected in sec- tors of fourth-whorl staminoid organs (Figures 2V and 2W), with the level of signal being usually lower than that found in the same tissues of second- and third-whorl organs.

\section{The Tdef Phenotype Is Dependent on PI and Is Masked by floto}

The results discussed above suggest that $D E F$ is expressed and can influence organ identity in Arabidopsis. To determine which genes are required for the Tdef phenotype, the TDF1-1 plant was crossed to $\mathrm{Pi}$ and Flo10 mutant backgrounds. $p i-1$ is a recessive nonsense allele that results in a relatively strong phenotype (Bowman et al., 1989; Goto and Meyerowitz, 1994). Pi-1 flowers have sepaloid second-whorl organs and third-whorl organs that are freestanding filamentous structures or carpelloid organs fused to the fourthwhorl carpels. $F_{2}$ progeny homozygous for the pi-1 allele and carrying the $D E F$ transgene were identified genetically, and their floral phenotype was characterized. These $\mathrm{Pi}-1 /$ Tdef plants had only $\mathrm{Pi}-1$ flowers, suggesting that $D E F$ cannot complement a pi mutation and that the Tdef fourth-whorl phenotype requires $P I$ function. Indeed, TDF1-1 plants heterozygous for the pi-1 allele had primarily wild-type flowers, with only the latest flowers showing a weak Tdef fourthwhorl phenotype, suggesting that this phenotype is sensitive to the level of PI protein in the plant.

A mutation in the FLO10 gene results in a replacement of the fourth gynoecial whorl by up to 10 stamens, with the average number of extra stamens decreasing in later flowers to 3.1 (Schultz et al., 1991; Bowman et al., 1992). Introducing $D E F$ into a flo10-1 background had no significant effect on the number of fourth-whorl staminoid organs of early and later flowers, compared with Flo10 plants (data not shown). Flo10 is therefore epistatic to the Tdef phenotype, distinguishing this case from experiments in which expressing Arabidopsis class $\mathrm{B}$ genes under a constitutive promoter

Figure 2. (continued).

(A) to (O) TDF flowers probed with DEF antisense RNA probe. (A) to (E) show TDF1 flowers at early stages of flower development. A clear DEF signal is first noticeable in the central dome of a stage 5 flower ([E], arrow). (F) to (I) are serial sections through stage 3 , 6 , and 7 flowers. No signal can be detected in the stage 3 flower. DEF expression is seen in third-whorl stamen primordia and in fourth-whorl organs. Arrows point to fourth-whorl cells expressing DEF transcript. $(\boldsymbol{J})$ and $(\mathbf{K})$ show stage 11 flowers. Notice that the fourth whorl consists of both stamen and carpel tissue fused together and that the DEF transcript is located mostly in stamen and petal tissue. (L) to (O) are serial sections through a stage 7 flower. Notice that the primordia of second, third, and fourth whorls do not display uniform expression patterns of $D E F$.

(P) to (S) TDF flowers probed with Pl antisense RNA probe. (P) shows a stage 3 flower with $P$ / expression in the central dome similar to that of the wild type. (Q) to (S) show sections through stage 5, 7, and 9 flowers. $P /$ is expressed in second-and third-whorl primordia as occurred in the wild type. In addition, ectopic expression in fourth-whorl organs can clearly be seen (arrows).

(T) to (W) TDF flowers probed with AP3 antisense RNA probe. (T) and (U) show sections through stage $3,4,5$, and 7 flowers. AP3 is expressed in second-and third-whorl primordia as occurred in the wild type. Notice in (U) that abnormal stamen-carpel primordia (asterisk) in the fourth whorl are devoid of $A P 3$ signal. (V) shows a stage 7 flower in which the AP3 transcript is found in cells of abnormal fourth-whorl organ primordia (arrow). Unlike this flower, most Tdef flowers showed a pattern similar to that shown in (U). (W) shows a stage 9 flower with staminoid fourthwhorl organs. The AP3 transcript is seen in third-whorl stamens and in a limited number of cells (asterisk) in the fourth whorl.

I, first-whorl organ; II, second-whorl organ; III, third-whorl organ; IV, fourth-whorl organ; C, carpel; P, petal; S, sepal; T, stamen. Numbers refer to stages of floral development. Magnifications are as follows: (A) $\times 417 ;(B) \times 282 ;(C) \times 467 ;(D) \times 350 ;(E) \times 310 ;(F)$ to (I) $\times 150 ;(J)$ and (K) $\times 81$; (L) to (O) $\times 262 ;(P) \times 367 ;(\mathbf{Q}) \times 350 ;(R) \times 236$; (S) $\times 158 ;(T) \times 148 ;(U) \times 147 ;($ V) $\times 121 ;(W) \times 147$. 
caused an enhancement of the Flo10 phenotype (Krizek and Meyerowitz, 1996a).

\section{The DEF Gene Complements ap3 Mutations}

To determine whether DEF can functionally replace its putative Arabidopsis homolog AP3, crosses were made between TDF1-1 and both Ap3-1 and Ap3-3 plants. ap3-1 is a temperature-sensitive, recessive allele resulting from a missense mutation in the K-box. The Ap3-1 phenotype is relatively weak, with sepaloid organs in the second whorl and typically freestanding carpelloid stamens in the third whorl when the plant is grown at $22^{\circ} \mathrm{C}$ (Bowman et al., 1989). ap3-3 is a recessive allele resulting from a nonsense mutation in the first exon that results in a relatively strong phenotype similar to pi-1 (Figure 3A; Jack et al., 1992). $F_{2}$ progeny homozygous for the ap3 mutant alleles and carrying the DEF transgene (Ap3/Tdef) were identified genetically, and the floral phenotype was characterized. All Ap3/Tdef plants produced flowers that were intermediate in phenotype between Ap3 and Tdef flowers (Figures $3 \mathrm{~B}$ and $3 \mathrm{C}$ ). These modified Tdef flowers had petals or sepal-petal organs in the second whorl, normal stamens or carpel-stamens in the third whorl, and stamens or stamen-carpels in the fourth whorl. The floral phenotype varied, depending on the position of the flower on the inflorescence, so that as one moves along the inflorescence in an acropetal direction, the degree of fourth-whorl staminody and second- and third-whorl complementation increases. Later flowers had some completely normal petals and stamens in the second and third whorl (Figure 3D). These results show that the DEF gene can replace the AP3 gene in making normal petals and stamens and can also cause the Tdef fourth-whorl phenotype in the absence of $A P 3$ activity. The degree to which DEF can complement depends on the position of the flower in the inflorescence. The TDF3 line, which had no floral phenotype, could not complement even the weak ap3-1 allele (data not shown).

\section{Maintenance of the AP3 Transcript Is Independent of Tissue Type and Dependent on the AP3 Protein}

As shown in the previous section, DEF can replace AP3 in making normal petals and stamens. We also wanted to determine whether $D E F$ could replace $A P 3$ in the maintenance of AP3 transcript levels. For this purpose, we looked at the distribution of AP3 transcript in Ap3-3, Pi-1, and Ap3-3/Tdef plants. As previously described (Goto and Meyerowitz, 1994; Jack et al., 1994), the pattern of AP3 transcript distribution in both mutants (Ap3-3, Figures $4 \mathrm{~A}$ and $4 \mathrm{~B}$; Pi-1, Figures $4 C$ and $4 D$ ) is similar to that in the wild type until stage 5. At later stages, continued high expression of the $A P 3$ transcript in a subset of cells at the base of the first and second organs can clearly be seen. Thus, unlike in previous reports (Goto and Meyerowitz, 1994; Jack et al., 1994), we could not see a clear difference in AP3 expression be-
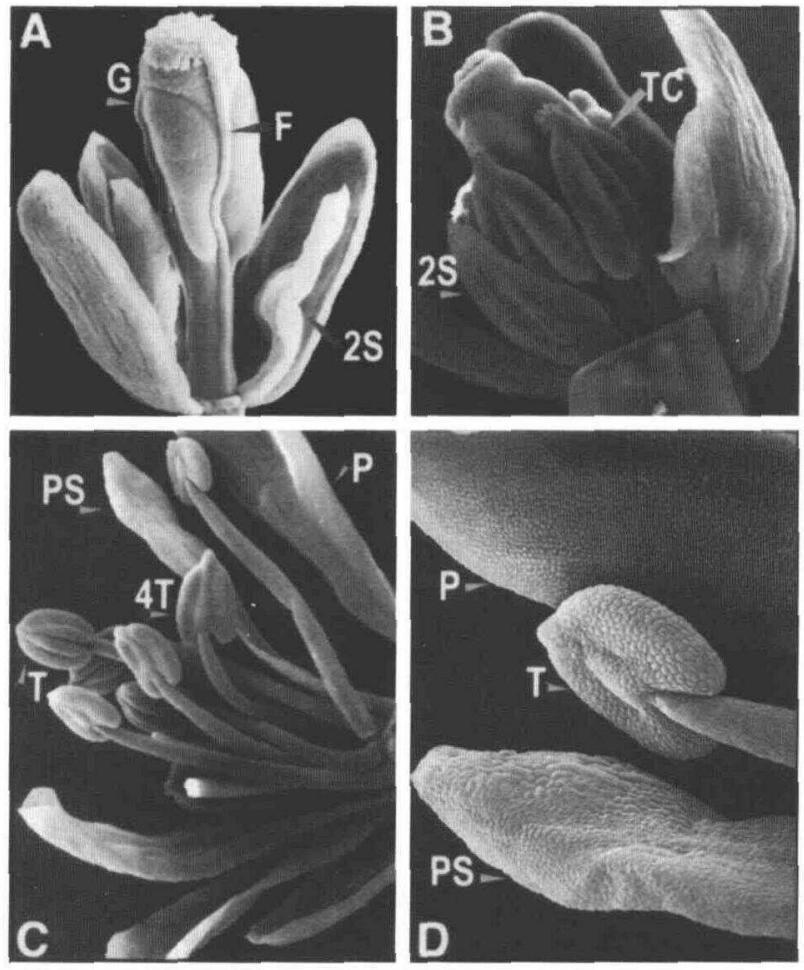

Figure 3. Scanning Electron Microscopy of Ap3-3 Flowers Complemented by $D E F$.

Some perianth organs were removed to show more clearly the reproductive organs.

(A) An Ap3-3 flower with second-whorl sepaloid organs (2S) and third-whorl filaments $(F)$ fused to the fourth-whorl gynoecium (G). Magnification is $\times 20$.

(B) An Ap3-3/Tdef flower with second-whorl sepaloid organs and third-whorl stamens with stigmatic papillae (TC).

(C) An Ap3-3/Tdef flower with second-whorl petals $(P)$, secondwhorl petals with sectored sepaloid tissue (PS), third-whorl stamens $(\mathrm{T})$, and additional fourth-whorl stamens (4T) replacing the gynoecium. Magnification is $\times 16$.

(D) Detailed view of the section from (C), showing second-whorl petals (P), petal-sepals (PS), and normal third-whorl organs ( $T$ ). Magnification is $\times 53$.

tween the two class B mutants. Our data suggest that some cells do not require functional class $B$ proteins to continually express the AP3 transcript.

The expression pattern of ap3-3 in most Ap3-3/Tdef flowers (Figures 4E to $4 \mathrm{~L}$ ) was similar to that seen in Ap3-3 flowers. At late stages of flower development, the transcript was clearly seen in a subset of cells in developing petals but not in the developing stamens (Figures $4 \mathrm{G}$ to $4 \mathrm{I}$ ). Morphologically normal stamens devoid of ap3-3 transcript (Figure 4J) developed. Rarely were third-whorl stamens showing limited sectors of ap3-3 expression found (Figure $4 \mathrm{~J}$ ). These results suggest that $D E F$ and $P I$ activity is not sufficient to maintain normal $A P 3$ transcription in the absence of functional $A P 3$ gene product. 

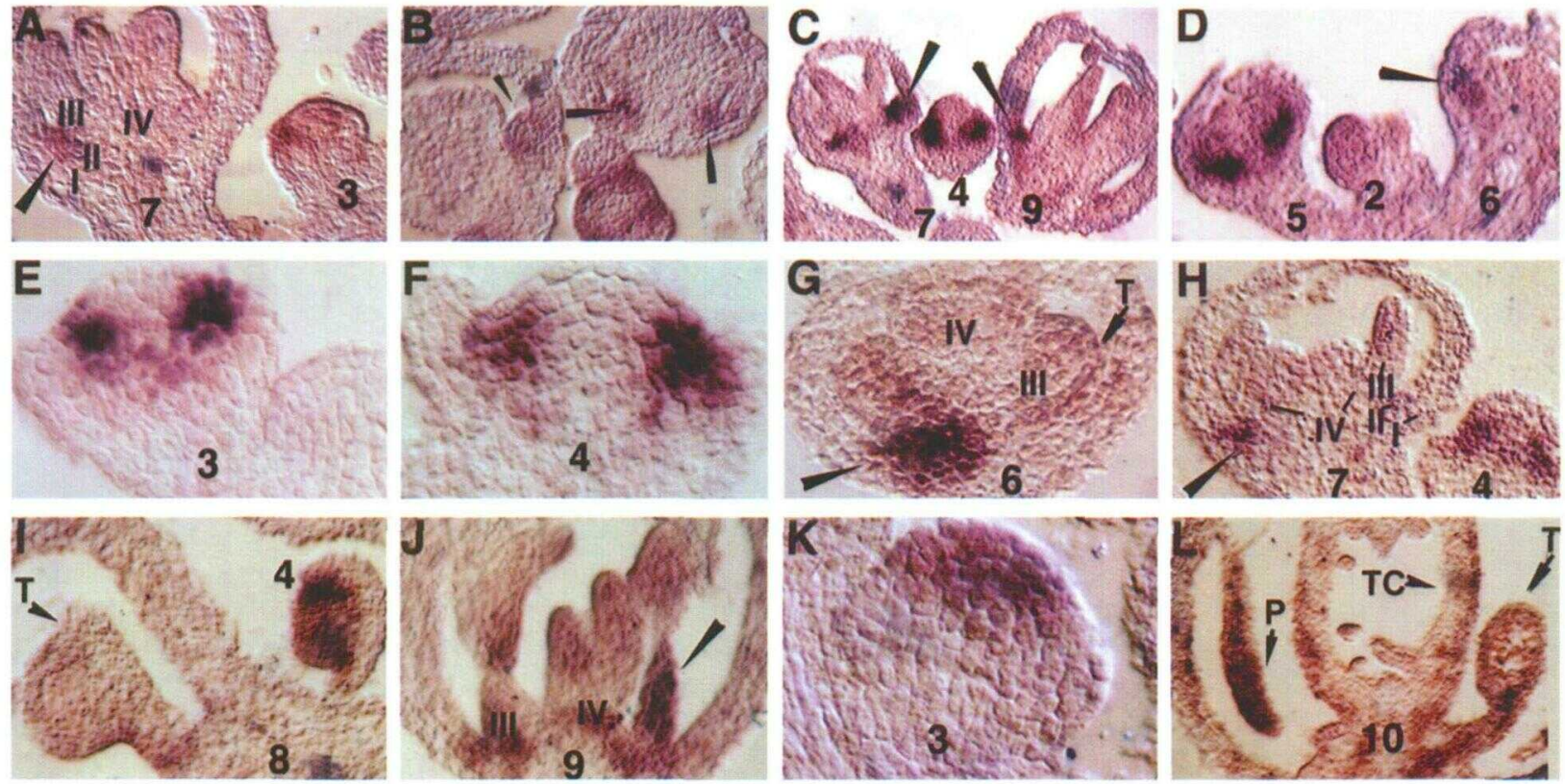

Figure 4. Distribution of $A P 3$ and $P I$ mRNA in Mutant Backgrounds.

Sections were hybridized with digoxigenin-labeled antisense AP3 ([A] to [J]) or PI ([K] and [L]) RNA probes and photographed under differential interference contrast optics. The transcript signal is dark brown. All sections except for the section shown in (B) (transverse) are longitudinal.

(A) and (B) Ap3-3 inflorescences. Expression of $A P 3$ is similar to that of the wild type in a stage 3 flower. Mature flowers maintain the $A P 3$ transcript in a subset of cells (arrowheads).

(C) and (D) $\mathrm{Pi}-1$ inflorescences. Expression of $A P 3$ is similar to that of the wild type in a stage 4 flower. Mature flowers maintain the AP3 transcript in a subset of cells (arrowheads), as occurred in AP3-3 inflorescences.

(E) to (J) Ap3-3/Tdef inflorescences probed with the AP3 antisense RNA probe. Expression of AP3 is similar to that of the wild type in stage 3 and stage 4 flowers. Mature flowers maintain the AP3 transcript in a subset of cells (arrowheads), as occurred in AP3-3 inflorescences. In (I), notice a stage 8 flower with a developing stamen devoid of AP3 expression. A stage 4 flower with signal is shown beside it for comparison. In ( $\mathrm{J}$ ), notice that in this stage 9 flower, AP3 transcripts are also seen in a subset of cells of the third-whorl stamens (arrowhead). Unlike this flower, most Ap3-3/Tdef flowers showed a pattern similar to (I).

(K) and (L) Ap3-3/Tdef inflorescences probed with the PI antisense RNA probe. In (K), a stage 3 flower shows normal expression of $P$ I in the central dome of the flower primordia. A mature flower with normal Pl expression in petals and stamens is shown in (L).

I, first-whorl organ; II, second-whorl organ; III, third-whorl organ; IV, fourth-whorl organ; P, petal; T, stamen; TC, staminoid-carpel. Numbers refer to stages of floral development. Magnifications are as follows: (A) $\times 150$; (B) $\times 148$; (C) $\times 90$; (D) $\times 144$; (E) $\times 365$; (F) $\times 378$; (G) $\times 284$; (H) $\times 160$; (I) $\times 136$; (J) $\times 81$; (K) $\times 396$; (L) $\times 91$.

PI transcript was not detected in developing organs of an Ap3-3 mutant (Goto and Meyerowitz, 1994). The DEF gene could successfully replace $A P 3$ in restoring normal $P I$ expression in the second- and third-whorl organs (Figures $4 \mathrm{~K}$ and $4 \mathrm{~L})$.

\section{DISCUSSION}

\section{Comparison of the DEF and AP3 Genes in a Common Genetic Background}

The transfer of floral regulators from one plant species to a distantly related one has provided important information on gene function. In previous studies, a cDNA clone under the control of either a constitutive viral promoter (Mandel et al., 1992; Weigel and Nilsson, 1995; Davies et al., 1996b) or a promoter from the recipient species (Irish and Yamamoto, 1995) was used as a transgene. In contrast, we have compared the function of two analogous class B organ identity genes from diverse angiosperm species by introducing them into the same genetic background. A genomic clone of the Antirrhinum class $B$ organ identity gene DEF complete with its endogenous promoter was introduced into Arabidopsis, and its expression and function were compared with that of the Arabidopsis AP3 gene in wild-type and mutant backgrounds. Like $A P 3$, transcription of the DEF gene in Arabidopsis is floral specific and maintained at high levels in petal and stamen primordia. The DEF protein can restore normal 
petal development in whorl two and stamen development in whorl three of strong Ap3 mutant flowers. Like $A P 3, D E F$ function depends on a functional product made by the second Arabidopsis class B organ identity gene PI. Thus, for the most part, the $D E F$ gene functions like the $A P 3$ gene. However, there were also differences between $A P 3$ and $D E F$ function and expression in Arabidopsis. Unlike $A P 3, D E F$ is also expressed in fourth-whorl primordia, where it promotes $P I$ expression and development of stamen tissue. DEF is also incapable of substituting for $A P 3$ in maintaining $A P 3$ transcript. In the following discussion, we propose that both of these differences result from the fact that the $D E F$ gene has regulatory elements more similar to the $P /$ gene than to the AP3 gene.

\section{Comparison of DEF Expression and Function in Arabidopsis Versus Antirrhinum}

Both $D E F$ in Antirrhinum and $P I$ in Arabidopsis are expressed initially in the second-, third-, and fourth-whorl floral primordia of stage 3 and stage 4 wild-type flowers. Expression of these genes in the fourth whorl is transient because their class B partners, required for transcript maintenance and protein stability, are absent from this tissue. Our data are consistent with the idea that the DEF gene in Arabidopsis is regulated, as it is in Antirrhinum, with an expression pattern more similar to $P /$ than to AP3. In Tdef Arabidopsis plants, initial expression of both $D E F$ and $P I$ in the fourth whorl enables persistent expression of both genes in this tissue and homeotic transformation of carpels to stamens. DEF expression in Arabidopsis differs from its expression in Antirrhinum in two ways. Unlike Antirrhinum, we can only detect clear expression of the DEF gene in Tdef plants when second- and third-whorl primordia begin to form (stage 5 flowers). Although expression in early stages was not detectable above background levels by in situ hybridization, we suspect that sufficient functional product was made by stage 4. That is because others have shown that $A P 3$ is required in these early stages (Bowman et al., 1989), and we know that $D E F$ can successfully replace $A P 3$. A simple explanation for the difference in levels of expression is that the efficiency of the DEF promoter in Arabidopsis is lower. We cannot rule out that not all regulatory elements required for $D E F$ expression are included in the genomic fragment used.

In Tdef and Ap3/Tdef inflorescences, as one moves along the inflorescence in an acropetal direction, the degree of fourth-whorl staminody and second- and third-whorl complementation increases. This observation suggests that in Arabidopsis, DEF function increases throughout inflorescence development and that such an increase can overcome any deficiencies in the intrinsic ability of DEF protein to function. Similar correlations between floral phenotype and position on the inflorescence have been noted for a wide variety of floral homeotic mutants (reviewed in Haughn et al., 1995; see also Krizek and Meyerowitz, 1996a) and are consistent with the idea that there is a gradual increase in the activation of genes in the floral program throughout inflorescence development.

\section{Maintenance of Class B Expression}

Previous studies have indicated that in the absence of functional class $B$ activity, class $B$ transcripts fail to be properly maintained in developing second- and third-whorl organs (Schwarz-Sommer et al., 1992; Tröbner et al., 1992; Goto and Meyerowitz, 1994; Jack et al., 1994; Zachgo et al., 1995). However, our results (Figure 4) and those of others (Goto and Meyerowitz, 1994) clearly show that in Arabidopsis, expression of class $B$ transcripts persists in some cells of the developing second and third whorls of class B mutants. Typically, in class $B$ mutants, cells expressing $A P 3$ are found in the second whorl, whereas cells expressing $P l$ are located in the third whorl, as if such cells have been pushed aside by those proliferating to form the second-and third-whorl organs. Such data demonstrate that in some cells, class B activity is not required for class B gene expression in the later stages of Arabidopsis floral development. It is possible that the same phenomenon is true for Antirrhinum, but in that case, the cells that keep on expressing class $B$ genes in the mutant background are either dispersed or harder to notice because they occupy a relatively smaller part of much bigger organs.

A simple explanation for persistent class $B$ gene expression in some cells of class B mutants is as follows. The transcription factors that initiate class $B$ gene expression in cells of stage 3 flowers are capable of maintaining class $B$ gene expression as long as such cells do not divide. Division of these cells dilutes the initial factor to a point at which class $B$ expression is not maintained without the activation by a functional class $B$ heterodimer. There is increasing evidence that class B function promotes cell division (Tröbner et al., 1992; Krizek and Meyerowitz, 1996a). In wild-type flowers, cells initially expressing both AP3 and PI would both proliferate and continue to maintain class $B$ gene expression, ultimately giving rise to a domain occupying the second and third whorls. In Ap3 or Pi mutants, many of the cells initially expressing class $B$ transcripts may not divide. Such cells would continue to express class $B$ genes even in the $a b-$ sence of class $B$ heterodimer but be pushed aside by cells expressing only class $A$ or class $C$ organ identity genes that are actively proliferating.

\section{Ap3 and DEF Transcripts Are Maintained by Different Factors}

We have shown that in fourth-whorl stamens of Tdef plants and second-, third-, and fourth-whorl organs of Ap3/Tdef plants, the $A P 3$ transcript is not maintained. Only on rare occasions can patches of $A P 3$ expression be detected in these organs. How can the DEF protein replace the AP3 protein in 
activating petal and stamen development and yet not be capable of substituting for AP3 in the maintenance of $A P 3$ transcription? A simple explanation for these data would be that AP3 can bind to a site within its own promoter that DEF cannot recognize. We do not favor this hypothesis for two reasons. First, different MADS box proteins seem to bind similar CarG box sequences in vitro (Riechmann et al., 1996). Second, switching the DNA binding domain of AP3 with that of other MADS box proteins did not affect its function (Krizek and Meyerowitz, 1996b). The current model must be refined to take into account our results and the following recent observations. (1) Maintenance of class B transcript levels requires additional floral factors (Schwarz-Sommer et al., 1992; Krizek and Meyerowitz, 1996a). (2) Class B functional specificity is attributed to regions required for protein-protein interactions (Krizek and Meyerowitz, 1996b). (3) Sequence comparisons suggest that it is unlikely that DEF would be able to replace PI more successfully than would AP3 in interactions with other proteins. (4) A sequence comparison of the $D E F$ and $A P 3$ promoters shows no similarities besides the CarG box (Irish and Yamamoto, 1995).

A revised model for maintenance of class B gene expression that is consistent with all of the information presented above is presented in Figure 5. We suggest that class B proteins indeed bind to their own promoters as heterodimers, yet maintenance of transcript levels requires an additional interaction of one of the class $B$ proteins with a third DNA binding transcription factor (DNA binding protein). Different factors would be required for $A P 3$ and $P I$ maintenance. $A P 3$ maintenance would require a factor that binds only to the $A P 3$ promoter at a site near the CarG box and interacts specifically with the AP3 protein (Figure 5A). A different factor, required for $P I$ maintenance, would bind to a site in the $P I$ promoter and interact with the PI protein. In Tdef transgenic plants (Figure 5B), the DEF protein cannot interact efficiently with the Arabidopsis AP3-specific factor. The DEF transcript is expressed and maintained in Arabidopsis in a pattern similar to $P I$. We predict that the $P l$-specific factor recognizes and binds to a site in the $D E F$ promoter and interacts with $\mathrm{PI}$ protein to allow maintenance of $D E F$. Interestingly, a mutation (DEF-chlorantha) in the $D E F$ promoter close to the CarG box causes severely reduced DEF transcription. In support of our hypothesis, it was proposed that this mutation defines a target sequence of the additional factor required for maintenance of $D E F$ transcription (Schwarz-Sommer et al., 1992). Our model predicts that in addition to the class $B$ heterodimer recognition element, the $D E F$ and $P I$ promoters should have a second cis-acting element in common. Such a prediction can be tested easily once the $P /$ promoter sequence has been published.

The inability of $D E F$ to properly control expression from the AP3 promoter provides an explanation for results from Irish and Yamamoto (1995). These investigators showed that unlike the DEF genomic clone, an AP3-DEF cDNA fusion gene under the control of the $A P 3$ promoter was unable to produce any wild-type petals or stamens in an ap3-3 background. In this case, the lack of full complementation

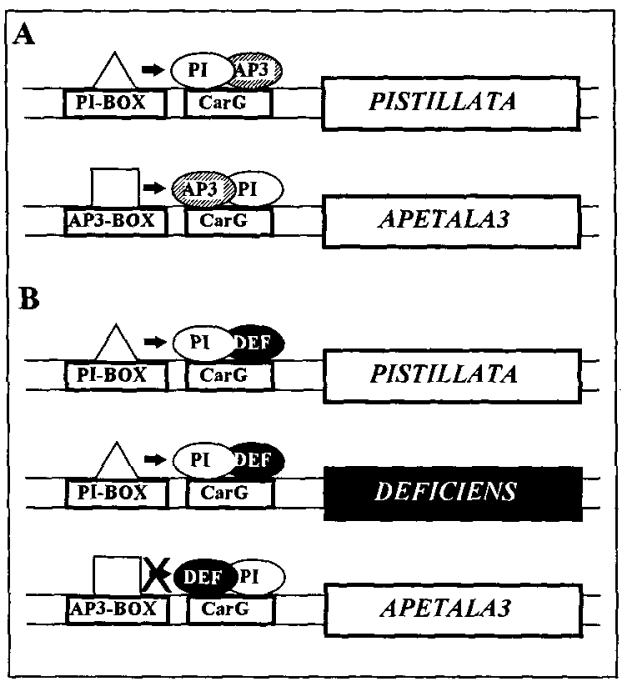

Figure 5. A Possible Mechanism for Class B Gene Maintenance.

The class $B$ genes, with their promoters and coding regions, are depicted schematically. cis-Acting binding sites are depicted as boxes in the promoter regions. Open, hatched, and black circles represent the PI, AP3, and DEF proteins, respectively.

(A) Autoregulation of class $B$ genes in wild-type Arabidopsis. Different factors bind to the $P I$ and $A P 3$ promoters (triangle and box, respectively). Each factor interacts specifically with just one of the class $B$ proteins of the heterodimer.

(B) Autoregulation of class B genes in transgenic Ap3-3/Tdef Arabidopsis plants. The $D E F$ gene is autoregulated by the $\mathrm{PI}$-specific factor. The AP3 transcript is not maintained because DEF cannot interact efficiently with the AP3-specific factor.

could be explained by the DEF protein's inefficiency in activating its own transcription via the $A P 3$ promoter.

$A$ direct association between the AP3 protein and $A P 3$ promoter and the DEF protein and DEF promoter has been suggested by in vitro binding experiments (Schwarz-Sommer et al., 1992; Tröbner et al., 1992; Riechmann et al., 1996). However, it has been difficult to rule out the possibility that in vivo class $B$ autoregulation is not direct but is controlled by a separate factor downstream of the class $B$ genes. We have shown here that developing stamen tissue can be devoid of the $A P 3$ transcript, providing in vivo evidence that autoregulation is not an indirect consequence of stamen development.

\section{Evolution of the Class B Expression Pattern}

In young flowers (stages 3 and 4 ) of both Arabidopsis and Antirrhinum, one of the class $B$ genes is expressed in the second-, third-, and fourth-whorl progenitor cells, whereas the other gene is expressed primarily in the second- and thirdwhorl progenitor cells. Expression of class $B$ genes in fourthwhorl organs has also been found in tobacco (Davies et al., 
1996b) and tomato (Pnueli et al., 1991, 1994). The DEF-like petunia gene $P M A D S 1$ appears to be an exception to this rule (Angenent et al., 1995), although spatial expression before late stage 4 has not been reported.

To date, there is no known function for class $B$ gene expression in the center of the young floral primordium. Indeed, somatic reversions of $D E F$ in the third whorl of Antirrhinum also restored fourth-whorl organs (Tröbner et al., 1992), suggesting that $D E F$ transcript is not required in the fourth whorl. How could such an expression pattern have evolved? The expression domain of class $B$ organ identity genes in ancestral angiosperm plants may have been broad and loosely regulated, with transcript levels declining gradually toward the center of the floral meristem, leading to the production of carpels. Such an expression pattern would result in flowers with many reproductive organs that vary in phenotype from functional stamen in the outermost whoris to functional carpels in the innermost whorls, with whorls of nonfunctional stamen-carpel organs in between, as has been observed in some members of the Magnoliaceae. A mutation in one of the class $B$ genes, causing its expression domain to be restricted to the second and third whorls, would result in a flower with only one whorl of stamens followed immediately by carpel formation, as has been observed in the simple complete flowers of Arabidopsis and Antirrhinum.

Oddly, the class B organ identity genes in Arabidopsis and Antirrhinum expressed primarily in the second and third whorls (AP3 and GLO) are not orthologous. This difference could be explained by the possibility that the restricted expression pattern of a class $B$ gene evolved more than once among the angiosperms after the progenitors of these two species were separated. Alternatively, the evolution of heterogeneous expression patterns could have occurred in a common progenitor, and later on, a recombination event (illegitimate) occurred between the $5^{\prime}$ ends of the two class $B$ genes, switching regulatory regions between them. Such a recombination event would result in a partially sterile plant with a Tdef-like phenotype (stamen-carpels in the fourth whorl); however, those of its progeny that were homozygous for either the parental or recombinant chromosomes would be perfectly normal. Therefore, this recombinant would not only represent a divergence in transcript patterns but could also be the beginning for the formation of new species (producing progenitors of Antirrhinum and Arabidopsis) because a cross between the parental plant and the recombinant would lead again to partial sterility. Our results suggest that the $D E F$ and $P I$ genes share common upstream regulatory elements, causing us to favor this second hypothesis.

\section{METHODS}

\section{Growth Conditions}

Arabidopsis thaliana plants were grown in 5-inch-diameter pots containing prepared soil mix (Terra-Lite Redi Earth; W.R. Grace \& Co. Can- ada Ltd., Ajax, Ontario, Canada) and then transferred to growth chambers at $22^{\circ} \mathrm{C}$ and continuous light (90 to $120 \mu \mathrm{E} \mathrm{m}^{-2} \mathrm{sec}^{-1}$ ).

\section{Plant Transformation and Strain Construction}

A 7-kb EcoRl genomic fragment from Antirrhinum contains the DEF gene with $\sim 4 \mathrm{~kb}$ upstream and $0.25 \mathrm{~kb}$ downstream of the transcribed sequences (Schwarz-Sommer et al., 1992). This fragment was cloned into the EcoRl site of the binary transformation vector RD1 (R. Datla, unpublished data). This vector contains a T-DNA that also encodes kanamycin resistance and glucuronidase (GUS) activity. The T-DNA was transformed into wild-type Arabidopsis $\left(\mathrm{T}_{1}\right)$ by using Agrobacterium tumefaciens-mediated transformation methods. TDF1 was a result of in-the-plant transformation (Katavic et al., 1994) in ecotype Columbia, and TDF2 and TDF3 were a result of root transformation (Valvekens et al., 1988) in the Landsberg erecta and RLD ecotypes, respectively.

TDF1 was outcrossed to Columbia, and one of the $F_{1}$ plants was designated TDF1-1. TDF1-1, segregating for two loci, was used in crosses to the following homozygous mutant strains by manual crosspolination: Ap3-1 and Pi-1 (Bowman et al., 1989; gifts of M. Koornneef, Wageningen Agricultural University, Wageningen, The Netherlands), Ap3-3 (Jack et al., 1992; gift of E. Meyerowitz, California Institute of Technology, Pasadena, CA), and Flo10-1 (Schultz et al., 1991). The resulting kanamycin-resistant $F_{1}$ plants were allowed to self-fertilize, and the $F_{2}$ plants were analyzed for floral morphology. Genotypes were confirmed by test crosses and polymerase chain reaction amplification (to detect DEF). Both loci of TDF1-1 produced independent plants with a strong Tdef phenotype.

\section{Scanning Electron Microscopy}

Samples were fixed, dried, coated, and dissected as described previously (Wilkinson and Haughn, 1995).

\section{RNA in Situ Hybridization}

Gene-specific antisense probes were prepared from pD793 for AP3 (digested with Bglll; Jack et al., 1992), pcPINX for PI (digested with Nsil; Goto and Meyerowitz, 1994), and a plasmid containing the 3' end of DEF cDNA (Sommer et al., 1990). Preparation, hybridization, and detection of ${ }^{35}$ S-labeled antisense RNA were done as given in Huijser et al. (1992). Preparation of digoxigenin-labeled probes was according to the Boehringer Mannheim nucleic acid labeling kit. Tissue was fixed in FAA (3.7\% paraformaldehyde, $5 \%$ acetic acid, and $50 \%$ ethanol), according to Huijser et al. (1992), and embedded in paraffin (Paraplast Plus; Sigma). Sections $(8 \mu \mathrm{m})$ were prepared using a microtome. Sections were transferred to slides pretreated with Vectabond (Dimension Labs, Mississauga, Canada), dried at $40^{\circ} \mathrm{C}$ overnight, and affixed to the slides by raising the temperature of the hot plate to $56^{\circ} \mathrm{C}$ for $4 \mathrm{hr}$.

The in situ hybridization protocol used was a modified procedure based on that of Coen et al. (1990) and G. Drews (personal communication). Paraffin was removed by immersing slides in $100 \%$ xylene, $50 \%$ xylene $-50 \%$ ethanol, and $100 \%$ ethanol for 5 min each. Sections were hydrated by immersion in $95,85,70,50$, and $25 \%$ ethanol and $\mathrm{H}_{2} \mathrm{O}$ for $5 \mathrm{~min}$ each, treated with $2 \times$ SSPE $(300 \mathrm{mM} \mathrm{NaCl}, 20$ $\mathrm{mM} \mathrm{NaH} \mathrm{PO}_{4}, 2 \mathrm{mM}$ EDTA, pH 7) at $70^{\circ} \mathrm{C}$ for $20 \mathrm{~min}$, and incubated for $20 \mathrm{~min}$ at $37^{\circ} \mathrm{C}$ with $1 \mu \mathrm{g} / \mathrm{mL}$ proteinase $\mathrm{K}$ in $100 \mathrm{mM}$ Tris- $\mathrm{HCl}$, pH 
8 , and $50 \mathrm{mM}$ EDTA. Slides were then dehydrated in $25,50,75,85$, 95 , and $100 \%$ ethanol for 5 min each and air dried at $52^{\circ} \mathrm{C}$ for $10 \mathrm{~min}$. Hybridization was done overnight at $52^{\circ} \mathrm{C}$ with a digoxigenin-labeled RNA probe (2 to $20 \mathrm{ng}$ ) in $100 \mu \mathrm{L}$ of hybridization buffer ( $10 \mathrm{mM}$ Tris$\mathrm{HCl}, \mathrm{pH} 7.5,1 \mathrm{mM}$ EDTA, $300 \mathrm{mM} \mathrm{NaCl}, 50 \%$ formamide, $7 \%$ dextran sulfate, $1 \times$ Denhardt's solution [ $1 \times$ Denhardt's solution is $0.02 \%$ Ficoll type $400,0.02 \%$ polyvinylpyrrolidone, $0.02 \% \mathrm{BSA}$ ], $500 \mu \mathrm{g} / \mathrm{mL}$ tRNA, and $250 \mu \mathrm{g} / \mathrm{mL}$ poly(A) RNA). Slides were washed in $2 \times$ SSC $(1 \times \mathrm{SSC}$ is $0.15 \mathrm{M} \mathrm{NaCl}, 0.015 \mathrm{M}$ sodium citrate) for $5 \mathrm{~min}$ and twice in $0.2 \times \mathrm{SSC}$ at $52^{\circ} \mathrm{C}$ for $30 \mathrm{~min}$.

Immunological detection of the hybridized probe was performed according to Coen et al. (1990), with a few modifications. Slides were covered for 20 min with $1 \mathrm{~mL}$ of $1 \%$ blocking reagent (Boehringer Mannheim) in $100 \mathrm{mM}$ maleic acid, $\mathrm{pH}$ 7, and $150 \mathrm{mM} \mathrm{NaCl}$. Slides were then covered for $30 \mathrm{~min}$ in $1 \mathrm{~mL}$ of buffer A (1\% BSA [Sigma], $0.3 \%$ Triton $\mathrm{X}-100$ [Sigma], $100 \mathrm{mM}$ Tris- $\mathrm{HCl}, \mathrm{pH} \mathrm{7.5}$, and $150 \mathrm{mM}$ $\mathrm{NaCl})$. The slides were incubated for $4 \mathrm{hr}$ with $1 \mathrm{~mL}$ of dilute $(1: 1200)$ antibody conjugate (Boehringer Mannheim) in buffer $A$, followed by two washes in buffer A (each for $20 \mathrm{~min}$ ).

For the color reaction, slides were immersed twice for $5 \mathrm{~min}$ in substrate buffer $(100 \mathrm{mM}$ Tris- $\mathrm{HCl}, \mathrm{pH} 9.5,100 \mathrm{mM} \mathrm{NaCl}$, and 50 $\mathrm{mM} \mathrm{MgCl}$ ) and incubated overnight with $0.5 \mathrm{~mL}$ of $0.34 \mathrm{mg} / \mathrm{mL}$ nitro blue tetrazolium salt and $0.175 \mathrm{mg} / \mathrm{mL} 5$-bromo-4-chloro-3-indolylphosphate $\rho$-toluidine salt in substrate buffer in the dark. The color reaction was stopped with $10 \mathrm{mM}$ Tris- $\mathrm{HCl}, \mathrm{pH} 8$, and $5 \mathrm{mM}$ EDTA, and slides were viewed before (brown color) or after (blue color) ethanol dehydration, $100 \%$ xylene immersion, and coverslip mounting with Enteilen (Merck). Sections were photographed under differential interference contrast optics by using a light microscope (Leitz DRB; Leica, Wetzlar, Germany) with Ektachrome 160 ASA film (Eastman Kodak).

\section{ACKNOWLEDGMENTS}

This work was supported by a Natural Sciences and Engineering Research Council (NSERC) of Canada Research Grant to G.W.H. and an NSERC postdoctoral fellowship to S.E.K. We thank Drs. Zsuzsanna Schwarz-Sommer and Hans Sommer for valuable discussion, gifts of the $D E F$ genomic clone, and technical assistance with in situ hybridization; Marilyn Martin for technical assistance; Dr. Elliot Meyerowitz for the Ap3-3 mutant line and plasmid pD793; and Dr. Detlef Weigel for plasmid pcPINX. We are also grateful to Mark Pidkowich, Tamara Western, and Drs. Zsuzsanna Schwarz-Sommer, Marcos Egea-Cortines, and Mark Wilkinson for critical reading of the manuscript.

Received November 27, 1996; accepted February 19, 1997.

\section{REFERENCES}

Angenent, G.C., Busscher, M., Franken, J., Dons, H.J.M., and Van Tunen, A.J. (1995). Functional interaction between the homeotic genes $f b p 1$ and $p M A D S 1$ during petunia floral organogenesis. Plant Cell 7, 507-516.

Bowman, J.L., Smyth, D.R., and Meyerowitz, E.M. (1989). Genes directing flower development in Arabidopsis. Plant Cell 1, 37-52.
Bowman, J.L., Smyth, D.R., and Meyerowitz, E.M. (1991). Genetic interactions among floral homeotic genes of Arabidopsis. Development 112, 1-20.

Bowman, J.L., Sakai, H., Jack, T., Weigel, D., Mayer, U., and Meyerowitz, E.M. (1992). SUPERMAN, a regulator of floral homeotic genes in Arabidopsis. Development 114, 599-615.

Coen, E.S., Romero, J.M., Doyle, S., Elliott, R., Murphy, G., and Carpenter, R. (1990). floricaula: A homeotic gene required for flower development in Antirrhinum majus. Cell 63, 1311-1322.

Davies, B., and Schwarz-Sommer, Z. (1994). Control of floral organ identity by homeotic MADS-box transcription factors. In Plant Promoters and Transcription Factors: Results and Problems in Cell Differentiation, Vol. 20, L. Nover, ed (Berlin: SpringerVerlag), pp. 235-258.

Davies, B., Egea-Cortines, M., de Andrade Silva, E., Saedler, H., and Sommer, H. (1996a). Multiple interactions amongst floral homeotic proteins. EMBO J. 15, 4330-4343.

Davies, B., Di Rosa, A., Eneva, T., Saedler, H., and Sommer, H. (1996b). Alteration of tobacco floral organ identity by expression of combinations of Antirrhinum MADS-box genes. Plant J. 10, 663-677.

Gaiser, J.C., Robinson-Beers, K., and Gasser, C.S. (1995). The Arabidopsis SUPERMAN gene mediates asymmetric growth of the outer integument of ovules. Plant Cell 7, 333-345.

Goto, $K_{i}$, and Meyerowitz, E.M. (1994). Function and regulation of the Arabidopsis floral homeotic gene PISTILLATA. Genes Dev. 8, 1548-1560.

Haughn, G.W., Schultz, E.A., and Martinez-Zapater, J.M. (1995) The regulation of flowering in Arabidopsis thaliana: Meristems, morphogenesis and mutants. Can. J. Bot. 73, 959-981.

Hill, J.P., and Lord, E.M. (1989). Floral development in Arabidopsis thaliana: Comparison of the wild-type and the homeotic pistillata mutant. Can. J. Bot. 67, 2922-2936.

Huijser, P., Klein, J., Lonnig, W.E., Meijer, H., Saedler, H., and Sommer, H. (1992). Bracteomania, an inflorescence anomaly, is caused by the loss of function of the MADS-box gene squamosa in Antirrhinum majus. EMBO J. 11, 1239-1249.

Ingram, G.C., Goodrich, J., Wilkinson, M.D., Simon, R., Haughn, G.W., and Coen, E.S. (1995). Parallels between UNUSUAL FLORAL ORGANS and FIMBRIATA, genes controlling flower development in Arabidopsis and Antirrhinum. Plant Cell 7, 1501-1510.

Irish, V.F., and Yamamoto, Y.T. (1995). Conservation of floral homeotic gene function between Arabidopsis and Antirrhinum. Plant Cell 7, 1635-1644.

Jack, T., Brockman, L.L., and Meyerowitz, E.M. (1992). The homeotic gene APETALA3 of Arabidopsis thaliana encodes a MADS box and is expressed in petals and stamens. Cell 68, 683-697.

Jack, T., Fox, G.L., and Meyerowitz, E.M. (1994). Arabidopsis homeotic gene APETALA3 ectopic expression: Transcriptional and posttranscriptional regulation determine floral organ identity. Cell 76, 703-716.

Katavic, V., Haughn, G.W., Reed, D., Martin, M., and Kunst, L. (1994). In planta transformation of Arabidopsis thaliana. Mol. Gen. Genet. 245, 363-370.

Krizek, B.A., and Meyerowitz, E.M. (1996a). The Arabidopsis homeotic genes APETALA3 and PISTILLATA are sufficient to provide the $B$ class organ identity function. Development 122, 11-22. 
Krizek, B.A., and Meyerowitz, E.M. (1996b). Mapping the protein regions responsible for the functional specificities of the Arabidopsis MADS domain organ-identity proteins. Proc. NatI. Acad. Sci. USA. 93, 4063-4070.

Levin, J.Z., and Meyerowitz, E.M. (1995). UFO: An Arabidopsis gene involved in both floral meristem and floral organ development. Plant Cell 7, 529-548.

Ma, H. (1994). The unfolding drama of flower development: Recent results from genetic analyses. Genes Dev. 8, 745-756.

Ma, H., Yanofsky, M.F., and Meyerowitz, E.M. (1991). AGL.1AGL6, an Arabidopsis gene family with similarity to floral homeotic and transcription factor genes. Genes Dev. 5, 484-495.

Mandel, M.A., Bowman, J.L., Kempin, S.A., Ma, H., Meyerowitz, E.M., and Yanofsky, M.F. (1992). Manipulation of flower structure in transgenic tobacco. Cell 71, 133-143.

McGonigle, B., Bouhidel, K., and Irish, V.F. (1996). Nuclear localization of the Arabidopsis APETALA3 and PISTILLATA homeotic gene products depends on their simultaneous expression. Genes Dev. 10, 1812-1821.

Okamoto, H., Yano, A., Shiraishi, H., Okada, K., and Shimura, Y. (1994). Genetic complementation of a floral homeotic mutation, apetala3, with an Arabidopsis thaliana gene homologous to DEFICIENS of Antirrhinum majus. Plant Mol. Biol. 26, 465-472.

Pnueli, L., Abu-Abeid, M., Zamir, D., Nacken, W., SchwarzSommer, Z., and Lifschitz, E. (1991). The MADS box gene family in tomato: Temporal expression during floral development, conserved secondary structures and homology with homeotic genes from Antirrhinum and Arabidopsis. Plant J. 1, 255-266.

Pnueli, L., Hareven, D., Broday, L., Hurwitz, C., and Lifschitz, E. (1994). The TM5 MADS box gene mediates organ differentiation in the three inner whorls of tomato flowers. Plant Cell 6, 175-186.

Riechmann, J.L., Krizek, B.A., and Meyerowitz, E.M. (1996). Dimerization specificity of Arabidopsis MADS domain homeotic proteins APETALA1, APETALA3, PISTILLATA, and AGAMOUS. Proc. Natl. Acad. Sci. USA 93, 4793-4798.

Sakai, H., Medrano, L.J., and Meyerowitz, E.M. (1995). Role of SUPERMAN in maintaining Arabidopsis floral whorl boundaries. Nature 378, 199-203.

Schultz, E.A., Pickett, F.B., and Haughn, G.W. (1991). The FLO10 gene product regulates the expression domain of homeotic genes $A P 3$ and $P I$ in Arabidopsis flowers. Plant Cell 3, 1221-1237.
Schwarz-Sommer, Z., Huijser, P., Nacken, W., Saedler, H., and Sommer, H. (1990). Genetic control of flower development by homeotic genes in Antirrhinum majus. Science 250, 931-936.

Schwarz-Sommer, Z., Hue, I., Huijser, P., Flor, P.J., Hansen, R., Tetens, F., Lönnig, W., Saedler, H., and Sommer, H. (1992). Characterization of the Antirrhinum floral homeotic MADS-box gene deficiens: Evidence for DNA binding and autoregulation of its persistent expression throughout flower development. EMBO J. 11, 251-263.

Simon, R., Carpenter, R., Doyle, S., and Coen, E. (1994). Fimbriata controls flower development by mediating between meristem and organ identity genes. Cell 78, 99-107.

Smyth, D.R., Bowman, J.L., and Meyerowitz, E.M. (1990). Early flower development in Arabidopsis. Plant Cell 2, 755-767.

Sommer, H., Beltrán, J., Huijser, P., Pape, H., Lönnig, W., Saedler, H., and Schwarz-Sommer, Z. (1990). Deficiens, a homeotic gene involved in the control of flower morphogenesis in Antirrhinum majus: The protein shows homology to transcription factors. EMBO J. 9, 605-613.

Tröbner, W., Ramirez, L., Motte, P., Hue, I., Huijser, P., Lönnig, W., Saedler, H., Sommer, H., and Schwarz-Sommer, Z. (1992). GLOBOSA: A homeotic gene which interacts with DEFICIENS in the control of Antirrhinum floral organogenesis. EMBO J. 11, 4693-4704.

Valvekens, D., Van Montagu, M., and Van Lijesbettens, M. (1988). Agrobacterium tumefaciens-mediated transformation of Arabidopsis thaliana root explants by using kanamycin selection. Proc. Natl. Acad. Sci. USA 85, 5536-5540.

Weigel, D., and Meyerowitz, E.M. (1993). Activation of floral homeotic genes in Arabidopsis. Science 261, 1723-1726.

Weigel, D., and Meyerowitz, E.M. (1994). The ABCs of floral homeotic genes: Review. Cell 78, 203-209.

Weigel, D., and Nilsson, O. (1995). A developmental switch sufficient for flower initiation in diverse plants. Nature 377, 495-500.

Wilkinson, M.D., and Haughn, G.W. (1995). UNUSUAL FLORAL ORGANS controls meristem identity and organ primordia fate in Arabidopsis. Plant Cell 7, 1485-1499.

Zachgo, S., de Silva, E.A., Motte, P., Tröbner, W., Saedler, H., and Schwarz-Sommer, Z. (1995). Functional analysis of the Antirrhinum floral homeotic DEFICIENS gene in vivo and in vitro by using a temperature-sensitive mutant. Development 121, 2861-2875. 


\section{Divergence of Function and Regulation of Class B Floral Organ Identity Genes}

A. Samach, S. E. Kohalmi, P. Motte, R. Datla and G. W. Haughn PLANT CELL 1997;9;559-570

DOI: $10.1105 /$ tpc.9.4.559

This information is current as of August 17, 2010

Permissions

eTOCs

CiteTrack Alerts

Subscription Information
https://www.copyright.com/ccc/openurl.do?sid=pd_hw1532298X\&issn=1532298X\&WT.mc_id=pd_hw1532298X

Sign up for eTOCs for THE PLANT CELL at:

http://www.plantcell.org/subscriptions/etoc.shtml

Sign up for CiteTrack Alerts for Plant Cell at:

http://www.plantcell.org/cgi/alerts/ctmain

Subscription information for The Plant Cell and Plant Physiology is available at: http://www.aspb.org/publications/subscriptions.cfm 